\title{
INTUBATION USING LMA CTRACH IN ANAESTHETIZED BUT UNPARALYSED PATIENTS - A PILOT STUDY
}

Geetanjali Chilkoti, Medha Mohta, Anjali Kochhar, Deepti Agarwal, A K Sethi

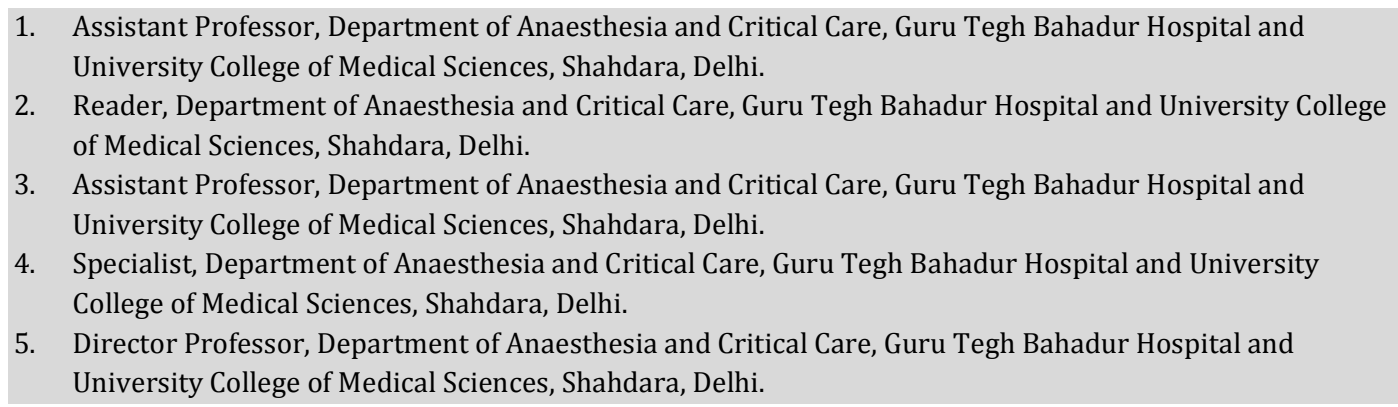

\section{CORRESPONDING AUTHOR}

Dr. Geetanjali Chilkoti, A-1404, Jaipuria Sunrise Greens,

Ahimsa Khand, Indirapuram, Ghaziabad

E-mail: geetanjalidr@yahoo.in,

Ph: 919868399782.

ABSTRACT: BACKGROUND - Laryngeal Mask Airway (LMA) CTrach is a supraglottic device which is functionally identical to Intubating Laryngeal Mask airway (ILMA) which enables ventilation and allows real time visualization of endotracheal intubation. This study aimed at assessing the ease and efficacy of using LMA CTrach for orotracheal intubation in anaesthetized but unparalysed patients. MATERIAL AND METHODS: 40 patients of ASA Grade I/ II scheduled for elective surgeries requiring orotracheal intubation were studied. Anaesthesia was induced with fentanyl $1 \mu \mathrm{g} . \mathrm{kg}^{-1}$ and sevoflurane. Neuromuscular blockers were not used. After CTrach insertion, various corrective manoeuvres were used to obtain the best laryngeal view. Two ml of $2 \%$ lignocaine was instilled over the vocal cords via a small sized catheter and tracheal intubation was performed after 2 minutes. The haemodynamic parameters were recorded. Any complications occurring following the CTrach insertion or tracheal intubation and those in the post operative period were also recorded. RESULTS: The insertion of CTrach was easy and well tolerated in all the patients. Thirty percent of patients required corrective manoeuvres. Tracheal intubation was successful in all 40 patients in first or second attempt. The problems encountered were hypotension following tracheal intubation in $20 \%$ patients and bucking over the tube at the time of CTrach removal in $17.5 \%$ patients. Hypotension persisted only for $2-3$ minutes. In the post operative period, mild sore throat and pain on swallowing were complained by four patients each, which resolved within 24 hours.

CONCLUSION - The use of LMA CTrach to ventilate lungs and facilitate tracheal intubation in anaesthetized unparalysed patients appears to be a useful and safe technique as it does not require patient cooperation and use of neuromuscular blockers.

KEY WORDS: Equipment - LMA CTrach; endotracheal intubation; anaesthetics - inhalation sevoflurane.

INTRODUCTION: Laryngeal Mask Airway (LMA) CTrach is a supraglottic device which is functionally identical to Intubating Laryngeal Mask airway (ILMA) but in addition has an 
integrated fibreoptic bundle with Liquid Crystal Display (LCD) 1, 2, 3. This system enables ventilation and allows real time visualization of endotracheal intubation ${ }^{2,3}$. Thus LMA CTrach can be a useful airway adjunct for management of difficult airway situation. It has been used for tracheal intubation after administration of neuromuscular blocking agents; awake insertion of the device followed by intubation using high concentrations of sevoflurane; or awake intubation using topical anaesthesia 1, 2, 4, 6, 8. All these methods have their own limitations. The administration of neuromuscular blocking agents may not be desirable in patients with anticipated difficult airway. High concentration of sevoflurane may lead to cardiovascular and cerebrovascular compromise in susceptible individuals ${ }^{7}$ and the procedure in awake patients cannot be performed without patient cooperation. If intubation can be performed through LMA CTrach using moderate concentrations of anaesthetic agents but without muscle paralysis, it would be a useful technique in uncooperative patients with anticipated difficult airway. As this airway device has never been used in such situations, the present study was conducted to assess the feasibility of using LMA CTrach in anaesthetized but unparalysed patients requiring orotracheal intubation for elective surgeries.

MATERIALS AND METHODS: After approval from the institutional ethics committee, 40 patients of American Society of Anaesthesiologists (ASA) Grade I/ II scheduled for elective surgeries requiring orotracheal intubation were studied. Informed consent was obtained from all the patients before inclusion in the study. Patients having limited mouth opening $\leq 25 \mathrm{~mm}$, oropharyngeal pathology or risk of regurgitation or pulmonary aspiration of gastric contents were excluded from the study.

The neck movements, Samsoon and Young modification of Mallampatti grading (MPG) and thyromental distance (TMD) were used to asses the airway of all the patients. The body mass index (BMI) and any other indicators of difficult airway, if present, were noted.

All the patients were kept fasting overnight and were given tab diazepam $10 \mathrm{mg}$ in the previous night and $5 \mathrm{mg}$ on the day of surgery 2 hours before anaesthesia. In the preanaesthetic room, inj. glycopyrrolate $0.2 \mathrm{mg}$ and Midazolam $1 \mathrm{mg}$ were administered intravenously. Standard intraoperative monitoring included continuous ECG, non-invasive blood pressure and pulse oximetry. Difficult airway cart was kept ready to manage any difficult airway situation. The LMA CTrach system was prepared after selecting appropriate size according to the manufacturer's recommendations (Size 3 for patients weighing $30-49 \mathrm{~kg}$, size 4 for $50-69 \mathrm{~kg}$ and size 5 for more than $70 \mathrm{~kg}$ ). Anaesthesia was induced with inj fentanyl $1 \mu \mathrm{g} \cdot \mathrm{kg}^{-1}$ and sevoflurane. Neuromuscular blockers were not used. The anaesthesia was considered adequate for CTrach insertion when the patient was unresponsive and the steady state end tidal concentration of $2 \%$ sevoflurane was achieved. The CTrach was inserted in the neutral head position by was introduced by one of the two senior anaesthesiologists who had the experience of using the airway device at least 20 times before the start of the study. The adequate spontaneous ventilation was confirmed by normal thoracic movements and capnography. After confirmation of the lung ventilation, the viewer was connected and the view of the laryngeal structures was noted according to the criteria listed in Table $1^{1}$. Whenever the laryngeal view was partial or grade II, various adjusting or corrective manoeuvres were used to improve the view depending on the likely cause of failure (Table 2) ${ }^{1}$. Three types of corrective manoeuvres used were Down-Up-Down (DUD), Medial-lateral-medial (MLM) and Chandy's manoeuvres.

After the vocal cords were visualized a small sized suction catheter was passed through the CTrach which could be easily seen on the viewer. Two $\mathrm{ml}$ of $2 \%$ lignocaine was instilled over the vocal cords through the catheter. After waiting for 2 min, a lubricated endotracheal tube was 
passed through the rigid anatomically curved airway tube of the CTrach.. During these two minutes, ventilation was assisted in all the patients. The correct tube placement was confirmed by direct visualization, chest auscultation and capnography. After the confirmation of the tracheal intubation, the CTrach was removed according to the manufacturer's instructions followed by the administration of neuromuscular blockers. The tracheal intubation was considered to have failed if trachea could not be intubated in three attempts. In these cases, it was decided to perform intubation by direct laryngoscopy using a Macintosh laryngoscope. In cases of failed direct laryngoscopy and intubation the plan was to use fibreoptic bronchoscope. Other rescue devices i.e. classic LMA and combitube were kept ready to deal with any "Can not intubate, Cannot ventilate " situation.

An independent observer noted the number of attempts of CTrach insertion and tracheal intubation, the time required to achieve ventilation through CTrach and the total time taken for the whole procedure. The time required to achieve ventilation was noted from the time of holding CTrach until the capnographic confirmation of adequate lung ventilation. The time taken for the whole procedure included the time required to achieve ventilation, optimize the laryngeal view, waiting period of 2 min for effective topical anaesthesia, tracheal intubation and removal of the CTrach. The ease of technique was also noted by the observer. It was assessed on a four point scale as easy, adjusting manoeuvers required, reinsertion required or failure at third attempt.

The heart rate, blood pressure and oxygen saturation were recorded before induction of anaesthesia, following CTrach insertion and after the tracheal intubation. Hypotension was defined as a fall in systolic blood pressure $\geq 20 \%$ of the preinduction value. Similarly a rise in systolic blood pressure more than $20 \%$ of the baseline value was defined as hypertension. Bradycardia and tachycardia were defined as heart rate less than 60 beats per minute and more than 100 beats per minute respectively.

Any complications occurring following the CTrach insertion or tracheal intubation e.g. coughing, bucking or bronchospasm; and those in the post operative period were recorded. The postoperative complications e.g. dysphagia, dysphonia, sore throat, nausea or vomiting were looked for and recorded at two and 24 hours after tracheal extubation.

RESULTS: We studied 40 patients, 11 males and 29 females, with mean age of $33.4 \pm 13.6$ yrs and weight of $54.7 \pm 12.0 \mathrm{Kg}$. The surgical procedures performed were laparoscopic cholecystectomy, ileostomy closure and incisional hernia repair. The mean (SD) duration of surgical procedures was $89.6 \pm 35.6$ min. There were 28,10 and 2 patients with modified MPG I, II and III respectively. Thyromental distance was within normal limit in all except three patients where it was less than $6 \mathrm{~cm}$. Out of these three patients, two patients were the same who had MPG III. In all these three patients the neck movements and mouth opening were adequate. The BMI of 36 patients were within normal limits whereas one patient had BMI $>35 \mathrm{Kg}^{-\mathrm{m}^{-2}}$ and the other three had BMI > $30 \mathrm{Kg} \cdot \mathrm{m}^{-2}$. The patient with BMI > $35 \mathrm{Kg} \cdot \mathrm{m}^{-2}$ also had reduced TMD with MPG III.

The LMA CTrach was easily inserted on first attempt and adequate ventilation could be achieved in all 40 patients. Only four patients had gag reflex during insertion which was self limiting. In $28(70 \%)$ patients, the initial laryngeal view was grade I, whereas it was grade II, III and IV in 6, 4 and 2 patients respectively. Corrective manoeuvres were required to obtain the optimum view of vocal cords in these $12(30 \%)$ patients. In 2 patients with immediate Grade IV view, secretions obscured the image for which the CTrach was removed, cleaned and reinserted. 
We were successful in performing tracheal intubation using CTrach in all 40 patients. In 39 patients the trachea was intubated in first attempt whereas in one patient two attempts were required inspite of grade I view of the vocal cords. The mean times to achieve ventilation and to conduct the whole procedure were $26.8 \pm 15.2 \mathrm{sec}$ and $4.3 \pm 0.6 \mathrm{~min}$ respectively. As far as the ease of technique is concerned, the technique was easy in 28 patients, corrective manoeuvres were required in 12 patients and reinsertion of the CTrach was needed in 2 patients.

Haemodynamic parameters of the patients are shown in table 3. Mean heart rate increased significantly after insertion of the LMA CTrach and also after endotracheal intubation when compared with the baseline heart rate. On the other hand, there was a significant decrease in systolic and diastolic blood pressures following CTrach insertion as well as endotracheal intubation in comparison to the pre induction values. Hypotension was detected in $4(10 \%)$ patients after CTrach insertion and in $6(20 \%)$ patients after endotracheal intubation. In all these patients hypotension persisted only for $2-3$ minutes and was managed by rapid infusion of intravenous fluid. All patients maintained oxygen saturation above $96 \%$ throughout the procedure.

There was no patient movement at the time of intubation through the CTrach, however, 7 (17.5\%) patients had bucking at the time of CTrach removal. These episodes of bucking were either self limiting or resolved following the administration of neuromuscular blockers. In the post operative period pain on swallowing and sore throat were complained by $4(10 \%)$ patients each. These complaints were managed conservatively and resolved within 24 hours.

DISCUSSION: This is the first reported series of the use of LMA CTrach in anaesthetized but unparalysed patients. The main advantage of this technique over awake tracheal intubation is that patient cooperation is not required and thus it can be easily used in uncooperative patients with anticipated difficult airway. This also eliminates the recall in the post operative period of intubation procedure.

For anticipated difficult airway situations, awake fibreoptic intubation remains the technique of choice ${ }^{6}$. However supraglottic devices are useful alternatives provided the mouth opening is adequate and there is no oropharyngeal pathology $10,11,12$. The CTrach provides a patent airway conduit 12,13 and the intubation can be facilitated under direct vision. The use of CTrach also gives the operator time to optimize the laryngeal view and patient's physiological parameters before intubation, as ventilation and oxygenation is continued throughout the intubation process $2,3,14$. Thus CTrach is a useful adjunct in difficult airway situations.

The performance evaluation studies of CTrach have shown the safety and efficacy of this device when used in paralysed patients with normal airway 1 , 4, 5. Liu et al reported $100 \%$ first attempt intubation success rate when CTrach was used in 84 normal paralysed patients ${ }^{4}$. It has also been successfully used in patients with potential $9,11,12$ or unanticipated difficult airway ${ }^{10}$.

The awake insertion of the CTrach followed by intubation under anaesthesia requires the inordinately high concentration of inhalational anaesthetic agents. Wender et al reported the awake insertion of CTrach in morbidly obese patients ${ }^{6}$. They attempted intubation once the end tidal concentration of sevoflurane reached 4-5\% for at least 1 min. Haemodynamic changes were not discussed in this case series. However, the administration of high concentrations of sevoflurane can cause cardiovascular or cerebrovascular compromise in susceptible patients ${ }^{7}$. In addition, awareness remains the problem during awake insertion of the LMA CTrach.

We could ventilate and intubate trachea successfully using CTrach in all 40 patients. Thus the ease of ventilation and success of intubation using CTrach are comparable in awake patients and anaesthetized but unparalysed patients. However, awake intubation using CTrach 
requires patient's cooperation and has the potential risk of recall and patient discomfort. Lopez et al reported the use of the LMA CTrach for awake intubation in 21 patients with difficult airway using sedation and topical instillation of lignocaine to anaesthetize the larynx ${ }^{8}$. They found CTrach easy to use for awake orotracheal intubation. However the incidence of recall was reported to be about 70\%. Also corrective manoeuvres were required in a large number of patients (50\%). In our series, only 30\% patients required these manoeuvres; however we had included all patients irrespective of airway classification. In all three patients (two patients with MPG III and reduced TMD and one patient with reduced TMD only) with anticipated difficult airway, we were able to ventilate and obtain the best laryngeal view following simple corrective manoeuvres ${ }^{1}$. In all these three patients we were able to intubate the trachea successfully in first attempt. Obesity, a risk factor for difficult mask ventilation, did not affect the ease of ventilation, requirement of corrective manoeuvres or the success of first attempt intubation in the present study.

In our study, hypotension was detected in $10 \%$ of patients after CTrach insertion and in $20 \%$ after endotracheal intubation. The cause of hypotension is definitely inhalational induction with sevoflurane as LMA insertion is attempted once the steady state end- tidal concentration of $2 \%$ sevoflurane is achieved. The comparative high incidence of hypotension after tracheal intubation can be attributed to the prolonged duration for which sevoflurane is used to maintain adequate depth of anaesthesia that include waiting period of two minutes following LA instillation over the vocal cords. Hypotension persisted only for short duration and was managed only by rapid infusion of intravenous fluid.

This study is the first series reporting the immediate and postoperative complications following the use of LMA CTrach. The only immediate complication encountered at the time of CTrach insertion was gag reflex in 4 patients which was self limiting. Bucking over the endotracheal tube at the time of removal of the CTrach was observed in 7 patients, although no such problem was encountered at the time of intubation through CTrach. This high incidence of bucking was probably due to carinal stimulation at the time of CTrach removal because of inadvertent inward movement of tracheal tube. As CTrach removal is a blind procedure, advancement of the tracheal tube is usual at this time as one tends to exert slight downward pressure on the tracheal tube so that it does not slip out at the time of removal of CTrach. This problem can be prevented by more appropriate designing of the CTrach endotracheal tube with a visual trigger or mark $3 \mathrm{~cm}$ proximal to the endotracheal cuff ${ }^{14}$. It would help placement of tracheal tube to appropriate depth within the trachea and thus prevent the stimulation of carina at the time of removal of CTrach.

Recently CTrach has been incorporated in an algorithm for difficult airway management. Amathieu et al prospectively assessed a difficult airway algorithm that included two modified optical devices i.e. Airtraq laryngoscope and LMA CTrach ${ }^{15}$. They restricted the use of awake intubation to only few indications like restricted mouth opening $\leq 25 \mathrm{~mm}$, severe cervical flexion deformity and history of previous impossible tracheal intubation. All the patients were anaesthetized and paralysed using succinylcholine or non depolarizing muscle relaxant. LMA CTrach was used for tracheal intubation following failed attempts with Macintosh and Airtraq laryngoscopes or as a rescue device in cases of impossible facemask ventilation. LMA CTrach achieved $100 \%$ success rate for ventilation and viewed tracheal intubation in this series.

LMA CTrach is available in four sizes i.e. 3, 4, 5 and 5L. Development of CTrach for paediatric patients is highly desirable as these are the potential candidates where awake intubation cannot be performed. 
CONCLUSION: The use of LMA CTrach for facilitating endotracheal intubation in anaesthetized but unparalysed patients provides a high success rate for both ventilation and tracheal intubation along with the advantages of avoidance of awareness and ease of use in uncooperative patients. We suggest incorporation of a visual trigger on the endotracheal tube to be used with LMA CTrach and development of paediatric sizes. We also advocate thorough training and clinical experience with this new optical device so as to increase the success of its use in difficult airway situations.

1. TABLE 1 Grading of vocal cord view ${ }^{1}$

\begin{tabular}{ll}
\hline Grade & View of laryngeal structure \\
\hline I & Full view of the arytenoids and glottis \\
III & Partial view of the arytenoids and the glottis opening \\
IV $\quad$ View includes dark areas or blurred view but identifiable structures \\
\end{tabular}

TABLE 2 Recommended adjusting manoeuvres to improve the grade of view ${ }^{1}$

\begin{tabular}{|ll|l}
\hline Grade of view & Adjusting manoeuvres & \\
\hline II & None & $\begin{array}{l}\text { Slightly shift the tip of the CTrach further } \\
\text { Increase light intensity on the viewer }\end{array}$ \\
III & $\begin{array}{l}\text { Perform suction through the channel for intubation } \\
\text { Try to position the tip of the CTrach below the epiglottis under direct vision ( } \\
\text { manoeuvres) }\end{array}$ & Down-up \\
IV & $\begin{array}{l}\text { Down-up manoeuvre in case of red out image } \\
\text { Reinsert the CTrach in case of white out image or Increase the light intensity on viewer } \\
\text { in black out image. }\end{array}$ & \\
\hline
\end{tabular}




\section{TABLE 3 Haemodynamic parameters; values are mean (SD)}

\begin{tabular}{llll|l}
\hline & Pre induction & After CTrach insertion & After intubation & \\
\hline $\begin{array}{l}\text { Heart rate } \\
\text { (bpm) }\end{array}$ & $94.5 \pm 17.2$ & $100.0 \pm 19.1^{*}$ & $106.0 \pm 18.1^{*}$ & \\
SBP (mmHg) & $124.4 \pm 14.2$ & $111.8 \pm 20.1^{*}$ & $107.1 \pm 17.7^{*}$ & \\
DBP (mmHg) & $79.7( \pm 9.3$ & $71.2 \pm 14.7^{*}$ & $69.9 \pm 14.9^{*}$ & \\
\hline
\end{tabular}

SBP: Systolic Blood Pressure, DBP: Diastolic blood pressure, "Significant difference from the pre induction values.

\section{REFERENCES:}

1 Timmerman A, Russo S, Graf B. Evaluation of the CTrach - an intubating LMA with integrated fiberoptic system. Br J Anaesth 2006; 96: 516-21.

2 Timmerman A, Russo S, Graf B. LMA CTrach ${ }^{\mathrm{TM}}$ : Initial experiences in patients with difficult to manage airways. Anaesthesia 2006; 55: 528-34.

3 Brain AIJ, Verghese C, Addy EV, Kapila A, Brimacombe J. The intubating laryngeal mask: a preliminary clinical report of a new means of intubating the trachea. Br J Anaesth 1997; 79: 704-9.

4 Liu EH, Goy R, Chen F. The LMA CTrach TM, a new laryngeal mask airway for endotracheal intubation under vision: evaluation in 100 patients. Br J Anaesth 2006; 96: 396-400.

5 Liu EH, Goy RW, Lim Y, Chen FG. Success of tracheal intubation with intubating laryngeal mask airways: a randomized trial of the LMA Fastrach and LMA CTrach. Anesthesiology 2008; 108: 621-6.

6 Wender R, Goldman AJ. Awake insertion of the fibreoptic intubating LMA CTrach in three morbidly obese patients with potentially difficult airways. Anaesthesia 2007; 62: 948-51.

7 Deem SA, Bishop JM, Bedford RF. Physiologic and pathologic responses to intubation. In: Hagberg CA, ed. Benumof's Airway management, $2^{\text {nd }}$ ed. Philadelphia: Mosby Elsevier 2007. pp. 193-214.

8 Lopez AM, Valero R, Pons M, Anglada T. Awake intubation using the LMA CTrach in patients with difficult airways. Anaesthesia 2009; 64: 387-91.

9 Dhonneur G, Ndoko SK, Yavchitz A, et al. Tracheal intubation of morbidly obese patients: LMA CTrach vs direct laryngoscopy. Br J Anaesth 2006; 97: 742-5.

10 Goldman AJ, Rosenblatt WH. The LMA CTrach in airway resuscitation: six case reports. Anaesthesia 2006; 61: 975-7.

11 Bjerkelund CE. Use of a new intubating laryngeal mask C Trach in patients with known difficult airway. Acta Anaesthesiol Scand 2006; 103: 508.

12 Goldman A, Rosenblatt W. Use of the fiberoptic intubating LMA CTrach TM in two patients with difficult airways. Anaesthesia 2006; 61: 601-3. 
13 Goldman A, Wender R, Goldman J. The LMA CTrach: a prospective evaluation of 100 cases. Anesthesiology 2006; 105: A 521.

14 Greenland KB. Fastrach TM tubes: modifying the design for use with the LMA CTrach тM? Br J Anaesth 2007; 99: 146-7.

15 Amathieu R, Combes X, Abdi W, Housseini LE, Rezzoug A, Dinca A et al. An algorithm for difficult airway management, modified for modern optical devices (Airtraque laryngoscope; LMA CTrach TM). Anesthesiology 2011; 114: 25-33. 